\title{
Cipango
}

Cipango Cahiers d'études japonaises

$17 \mid 2010$

La péninsule retrouvée

\section{Jean-Noël ROBERT (trad. et commentaires), La Centurie du Lotus, Poèmes de Jien (1155-1225) sur le Sūtra du Lotus}

Michel Vieillard-Baron

\section{(2) OpenEdition}

1 Journals

Édition électronique

URL : https://journals.openedition.org/cipango/1142

DOI : $10.4000 /$ cipango. 1142

ISSN : 2260-7706

\section{Éditeur}

INALCO

\section{Édition imprimée}

Date de publication : 30 juin 2010

Pagination : 273-277

ISBN : 978-2-85831-195-8

ISSN : $1164-5857$

\section{Référence électronique}

Michel Vieillard-Baron, « Jean-Noël robert (trad. et commentaires), La Centurie du Lotus, Poèmes de Jien (1755-1225) sur le Sūtra du Lotus », Cipango [En ligne], 17 | 2010, mis en ligne le 16 novembre 2012, consulté le 30 juin 2021. URL : http://journals.openedition.org/cipango/1142 ; DOI : https://doi.org/ 10.4000/cipango.1142

Ce document a été généré automatiquement le 30 juin 2021.

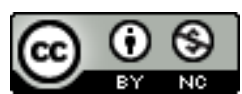

Cipango est mis à disposition selon les termes de la Licence Creative Commons Attribution - Pas d'Utilisation Commerciale 4.0 International. 


\title{
Jean-Noël ROBERT (trad. et commentaires), La Centurie du Lotus, Poèmes de Jien (1155-1225) sur le Sūtra du Lotus
}

\author{
Michel Vieillard-Baron
}

\section{RÉFÉRENCE}

Jean-Noël ROBERT (trad. et commentaires La Centurie du lotus : poèmes de Jien, 1155-1225 sur le «Sûtra du lotus », Paris: Collège de France, Institut des hautes études japonaises ; 2008, 1 vol. (XXV-266 p.) ; 24 cm. (Bibliothèque de l'Institut des hautes études japonaises).

Contient les 170 poèmes de Jien en japonais transcrit et trad. française à la suite. En appendice, contient les Cent deux citations de la version chinoise du « Sûtra du Lotus »et leurs traductions françaises, suivi de commentaire de Jien en japonais. Bibliogr. p. 217-224. Glossaire. ISBN 978-2-913217-19-5. $23 €$.

Qui connaît vraiment la poésie de Jien 慈円 (1155-1225) ${ }^{1}$ ? Avec quatre-vingt-douze waka retenus, Jien est, après Saigyō (quatre-vingt-quatorze pièces sélectionnées), le second poète le mieux représenté dans le Shinkokin wakashū 新古今和歌集, le « Nouveau recueil de poèmes anciens et modernes ", la huitième anthologie impériale, compilée sur ordre de l'empereur retiré Gotoba, achevée officiellement en 1205. Ce simple fait atteste la haute estime dont jouissait sa poésie, l'une des meilleures de son temps. La production poétique de Jien est considérable; son recueil personnel, le Shūgyoku shū 拾玉集 «Recueil des joyaux glanés », contient près de six mille pièces. Mais Jien n'était pas seulement poète. Il était également moine, et des plus éminents. Issu d'une famille puissante - son père et son frère furent grands chanceliers 
(kanpaku) -, Jien fit lui aussi une brillante carrière. Entré en religion à l'âge de douze ans, il fut, notamment, nommé à quatre reprises supérieur de la secte Tendai.

Dans son ouvrage, Jean-Noël Robert présente la traduction commentée de cent soixante-dix poèmes de Jien. Il s'agit de shakkyōka 釈教歌, c'est-à-dire de waka à thème bouddhique. Toutes ces pièces ont en outre pour caractéristique commune d'avoir été composées sur des thèmes extraits du Sūtra du Lotus, texte fondamental au sein de l'école Tendai. Les cent quarante-quatre premiers poèmes, composés sur cent deux citations extraites du Sütra, constituent « La Centurie du Lotus » proprement dite. JeanNoël Robert a complété ce premier corpus par deux autres ensembles: quatorze «Poèmes sur les dix ainsités » et douze "Poèmes japonais scripturaires sur les Dix "Ainsi » composés pour l'Assemblée estivale des Reliques ».

3 Comme le rappelle Jean-Noël Robert, Jien ne fut pas le premier à composer des poèmes japonais sur des thèmes extraits d'œuvres écrites en chinois ${ }^{2}$. Il ne fut pas non plus l'inventeur de la poésie à thème bouddhique, qui connut une certaine vogue dès la fin du $x^{e}$ siècle. En revanche, comme le souligne l'auteur, «nous lui devons sans doute la première centurie poétique consacrée au Lotus; cependant, et surtout, il était certainement le plus érudit en doctrine bouddhique de tous ceux qui avaient entrepris de versifier sur ce sūtra» (p. vI). C'est ainsi que, pour saisir pleinement le sens des poèmes traduits ici, une familiarité avec le waka classique ne suffit pas. Est également nécessaire une bonne connaissance du Sūtra du Lotus et de la doctrine de l'école Tendai. Afin de nous éclairer dans la lecture de ce corpus exigeant, on ne pouvait donc trouver guide plus compétent que Jean-Noël Robert qui, non seulement, est un spécialiste reconnu des doctrines de l'école Tendai, mais également un fin connaisseur du Sūtra du Lotus dont il a proposé une belle traduction française.

Dans l'Introduction, Jean-Noël Robert nous rappelle que «pour Jien, la composition poétique en langue japonaise n'était pas seulement une voie de salut à mettre au niveau de la voie bouddhique elle-même, mais qu'elle constituait en soi un exercice de méditation, les poèmes devenant le fruit concret de cette méditation» (p. XIX). C'est, ainsi, le produit d'une méditation sur la lettre du Sūtra du Lotus que nous sommes invités à lire.

5 Dans la traduction, comme dans la version originale, chaque poème est précédé de la citation du Sūtra qui lui sert de thème; Jean-Noël Robert présente ensuite la translittération du waka produit par Jien ${ }^{3}$, puis sa traduction. Cette traduction est suivie d'un commentaire détaillé dans lequel l'auteur précise les différentes connotations profanes et religieuses des termes employés par Jien ${ }^{4}$, ainsi que le sens du poème. Le lecteur se sent parfois pris de vertige devant l'écart entre sa compréhension du poème (le sens obvie) et, une fois que toutes les clefs lui ont été données, la signification véritable - doctrinale - de la pièce. Cependant, avec Jean-Noël Robert, le lecteur est entre de bonnes mains: l'érudition déployée dans les commentaires est impressionnante, sans jamais être pédante. Le style de l'auteur est élégant et précis, tant dans la traduction des poèmes que dans les gloses.

6 Pour illustrer de manière concrète notre propos, examinons deux exemples. Le premier est le poème $\mathrm{n}^{\circ} 22$ (dans la numérotation de J.-N. Robert) composé sur la citation "Savent que la Loi est à jamais sans nature »:

7 Sur ce thème Jien écrit : 
Naniwa-gata

fukaki e yori zo

nagarekeru

makoto wo shiru zo

mizu-guki no ato

Jean-Noël Robert traduit ce waka de la façon suivante :

\author{
Rive de Naniwa \\ de la baie profond \\ tel qu'il s'écoule \\ nous connaissons le vrai \\ par la trace des roseaux.
}

9 Quiconque connaît le waka classique aura repéré dans cette pièce des éléments familiers. Ainsi, la rive de Naniwa (située dans l'actuelle Ōsaka) est-elle un site célèbre chanté en poésie (uta-makura 歌枕), auquel on associe les roseaux. Ces derniers apparaissent ici de manière indirecte dans l'expression mizu-guki no ato «trace de la tige humide » - rendue dans la traduction par "trace des roseaux » - métaphore bien attestée pour l'écriture, qui désigne ici le Sūtra du Lotus. Après avoir glosé chacun des termes du poème, Jean-Noël Robert nous enseigne (p.39) que la pièce signifie également :

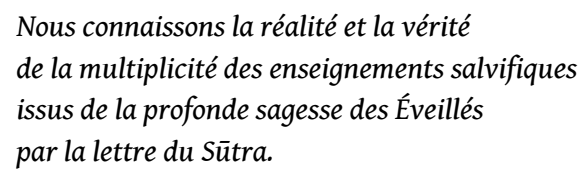

10 Une telle signification ne peut, bien évidemment, être saisie que par celui qui connaît parfaitement la doctrine bouddhique et la lettre du Sūtra. Nous sommes donc reconnaissants à Jean-Noël Robert de nous révéler ainsi des pans entiers de signification qui nous auraient échappés sans son commentaire, et qui donnent tout leur sens à la poésie de Jien.

11 Examinons à présent un second exemple. Il s'agit du poème $\mathrm{n}^{\circ} 33$ composé sur la citation du Sūtra : «L'amas de joyaux sans supérieur ». Sur ce thème Jien compose :

$$
\begin{aligned}
& \text { Toki shi areba } \\
& \text { motomenu hito mo } \\
& \text { kite zo miru } \\
& \text { yanagi-zakura no } \\
& \text { haru no nishiki wo }
\end{aligned}
$$

12 Poème que Jean-Noël Robert traduit :

$$
\begin{aligned}
& \text { Au temps venu } \\
& \text { même qui ne le recherche } \\
& \text { peut le contempler } \\
& \text { des saules et cerisiers } \\
& \text { le printanier brocart }
\end{aligned}
$$

Le lecteur distrait, pourrait prendre ce waka pour une simple pièce de printemps (il fait d'ailleurs allusion à un célèbre poème de printemps du Kokin wakashū 古今和歌集, la première anthologie impériale, compilée sur un ordre daté de 905), or, Jean-Noël Robert nous indique que «L'amas de joyaux » du thème désigne l'état d'Éveillé. Il nous signale en outre que la pièce de Jien fait allusion à la parabole de l'enfant pauvre qui, dans le Sūtra du Lotus, est narrée immédiatement après la citation choisie pour thème. Le premier vers «Au temps venu », résume, nous dit l'auteur, la période d'errance de l'enfant pauvre. « Le spectacle de la nature sert de métaphore à la révélation imprévue 
de la filiation élevée du fils prodigue, telle que la décrit la parabole du Sūtra " (p. 52-53). Enfin, le terme "brocart » du poème, correspond aux « joyaux » du thème. Comme le souligne Jean-Noël Robert, dans ce dernier poème «tous les rappels explicites de la lettre du Sūtra sont gommés. C'est au lecteur qu'est laissé le soin d'établir les rapprochements d'images et de sens avec la citation scripturaire» (p. 53). Selon les clefs données par l'auteur, la pièce de Jien signifie donc: Lorsqu'il en est temps, même celui qui ne le recherchait pas, peut atteindre l'état d'Éveillé.

Les deux exemples que nous venons de voir montrent l'exceptionnelle virtuosité de Jien qui, malgré les règles fort contraignantes et les conventions du waka, réussit à insuffler aux trente-et-une syllabes du poème un message religieux souvent dense et complexe. Jien, dont l'érudition en matière de religion était reconnue, place la barre très haut. On est en droit de se demander qui, en dehors de ses compagnons de l'école Tendai, pouvait, sans le moindre commentaire, comprendre ces pièces. Mais Jien ne composaitil pas avant tout pour la gloire du Bouddha, et pour lui-même? On aurait souhaité mieux connaitre le contexte de composition de ces poèmes. À quelle époque de la vie de Jien furent-ils produits? En quelle occasion? Dans quel objectif ? Pour quel public ? Ces informations auraient sans doute permis de mieux cerner la place de ces œuvres si particulières dans l'ensemble de la production de Jien. À la lecture de l'ouvrage, il ne fait toutefois aucun doute que ces waka sont des professions de foi. Comme le rappelle Jean-Noël Robert, pour Jien « ces poèmes japonais, qui sont vus par certains religieux comme le comble de la futilité, permettent en fait de communiquer avec la Voie réelle [du Sūtra du Lotus] » (p. xxII).

Jean-Noël Robert démontre à travers cet ouvrage que Jien est bel et bien un poète mystique ; il nous confirme en outre, plus largement, qu'au Japon, c'est sans doute dans le waka que s'exprime de la manière la plus éclatante l'union intime de l'homme avec la divinité.

\section{NOTES}

1. On connaît surtout aujourd'hui, semble-t-il, son travail d'historien. Jien est l'auteur du fameux Gukanshō 愚管抄 « Mes vues [sur l'histoire]» (début XIII siècle).

2. Dans un article qui a curieusement échappé à la vigilance de J.-N. Robert « Les Métamorphoses du mot: la citation de vers chinois comme sujet de composition de poèmes japonais, waka", Extrême-Orient, Extrême-Occident 17, Le travail de la citation en Chine et au Japon, 1995, p. 67-90, nous avons analysé l'évolution de ce mode de composition particulier, depuis l'origine, au $\mathrm{IX}^{\mathrm{e}}$ siècle, jusqu'à, précisément, l'époque de Jien (des poèmes de ce dernier sont d'ailleurs convoqués en guise d'exemples).

3. Le texte chinois des citations scripturaires et le texte japonais des waka figurent en fin d'ouvrage (p. 245-266).

4. L'auteur propose d'ailleurs en fin d'ouvrage (p. 225-244) un «Glossaire des termes littéraires japonais et de leur sens bouddhique chez Jien ». 